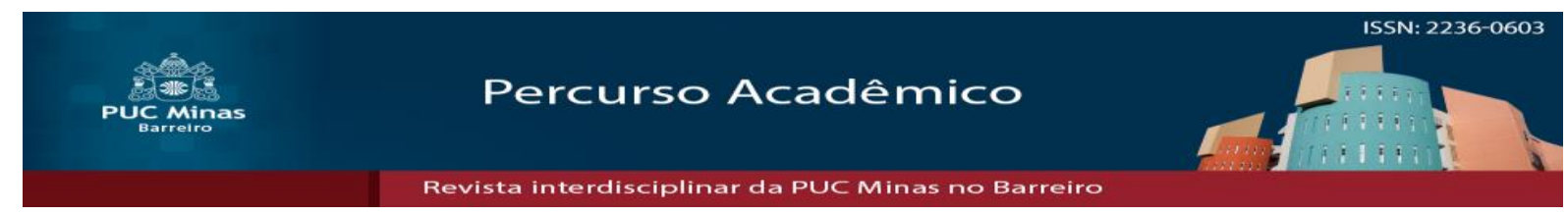

Dossiê: Saúde - Novas Tecnologias e Interlocuções - texto original (2) $(0)$

\title{
Análise da Capacitação "Caminhos do Cuidado": potenciais e desafios da formação de agentes comunitários de saúde e auxiliares e técnicos de enfermagem no campo de álcool e outras drogas
}

\author{
Analysis of the Inservice Training "Caminhos do Cuidado": potentials and \\ challenges of the education of community health workers, nurse's aides \\ and nursing technicians on the topic of alcohol and other drugs
}

\author{
Isabela Saraiva de Queiroz ${ }^{1}$ \\ Nathália Lopes Gomes Pinto Ferreira ${ }^{2}$
}

\begin{abstract}
Resumo
Este artigo tem por objetivo analisar de que modo a capacitação "Caminhos do CuidadoFormação em Saúde Mental (crack, álcool e outras drogas)", para agentes comunitários de saúde (ACS), auxiliares e técnicos de Enfermagem (ATEnf) das Equipes de Saúde da Família, pode contribuir na assistência ao usuáriode álcool e outras drogas. Analisou-se o material do curso com o objetivo de identificar potencialidades e limitações da proposta, considerando a formação e o trabalho dos profissionais. Verificou-se que o uso da metodologia ativa é um instrumento potente para o aprendizado dos ACS e ATEnf, por fomentar o papel ativo do aluno em sua aprendizagem e propiciar análise e reflexão sobre o tema do cuidado aos usuários de álcool e outras drogas.Entretanto, é necessário que a temática trabalhada na capacitação seja tratada de modo continuado, devido à sua complexidade, à existência de valores morais que impactam no trabalho e ao fato doparadigma da redução de danos ser relativamente recente no campo de trabalho com usuários de álcool e outras drogas.
\end{abstract}

Palavras-Chave: Capacitação em Serviço. Usuários de Drogas. Agentes Comunitários de Saúde. Enfermagem em Saúde Pública.

\begin{abstract}
This article aims to analyze how thein service training "Caminhos do Cuidado- Formação em Saúde Mental (crack, álcool e outras drogas)" for community health workers, nurse saides and nursing technicians of Family Health Strategy can contribute in assistance to drugusers. The material of the course has been analyzed to identify the potentials and the limits of the proposal, considering the workers practice and graduate. It was found that use active methodologyis a potent tool to the learning of community health workers, nurse saides and nursing technicians, because it fosters the active role of the student in their learning, and provide analysis and reflection on the subject of care to drug users. However, it's necessary that the theme displayed at the in service training be broached continuously, due the theme's complexity, the workers moral values and the fact that harm reduction policies are new in Brazil at the work with drugusers.
\end{abstract}

Keywords: In service Training. Drug Users. Community Health Workers. Public Health Nursing.

\footnotetext{
Artigo recebido em 05 de agosto de 2014 e aprovado em 27 de abril de 2016.

${ }^{1}$ Doutorado em Psicologia pela UFMG em 2015. Professor Adjunto IV da PUC-Minas. E-mail: isabelasq@gmail.com

${ }^{2}$ Psicóloga na Prefeitura Municipal de Belo Horizonte. Pós Graduanda em Saúde Mental. E-mail: nathlgpf@gmail.com
} 


\section{Introdução}

Neste artigo busca-se investigar como a capacitação "Caminhos do CuidadoFormação em Saúde Mental (crack, álcool e outras drogas)”,para agentescomunitários de saúde (ACS), auxiliares e técnicos de Enfermagem (ATEnf) das Equipes de Saúde da Família, pode contribuir na assistência ao usuário. Para isso, realizou-se análise documental do materialdo curso citado, apresentando-se os potenciais e os desafios da proposta.

\section{Sobre o curso}

O curso integra o eixo do cuidado do Plano "Crack é possível vencer" e tem por base a política do Ministério da Saúde para atenção integral a usuários de álcool e outras drogas.

O Plano citado foi lançado pelo Governo Federal, em 2011, visando à ampliação da oferta de serviços de tratamento e atenção ao usuário de crack e outras drogas, o combate ao tráfico de drogas e fomento de ações educativas sobre o tema.

As ações do Plano foram distribuídas em três tópicos:

1) Prevenção: visa ao fortalecimento de fatores de proteção e redução de fatores de risco para o uso de drogas. Também são ofertadas capacitações para profissionais de saúde, assistência social, educação, segurança pública, lideranças comunitárias e religiosas e operadores do Direito.

2) Autoridade: ações repressivas ao tráfico de drogas

3) Cuidado: desenvolvimento de ações de assistência para os usuários de álcool e outras drogas e seus familiares. Inclui ampliação da rede de cuidados e da oferta de capacitações para os trabalhadores de saúde e assistência social. (BRASIL, 2013, p.7).

O conteúdo do curso "Caminhos do Cuidado" foi dividido em três eixos:

1) O território, as redes de atenção, os conceitos, as políticas e as práticas de cuidado em saúde mental.

2) A caixa de ferramentas dos ACS e dos ATEnf na Atenção Básica.

3) A Reforma Psiquiátrica, a Redução de Danos e a Integralidade do Cuidado como diretrizes para intervenção em Saúde Mental e no uso de álcool, crack e outras drogas. 
A formação tem a carga horária de 60 horas, sendo 40 horas de atividades presenciais e 20 horas de atividades de dispersão. Estasse referem a momentos em que os participantes problematizam os conceitos aprendidos "in loco", por meio de pesquisas e intervenções no território em que trabalham, visando à identificação de elementos que traduzam a aplicação do conteúdo discutido nas oficinas, articulando teoria e prática.

\section{A metodologia ativa}

A metodologia do curso é ativa e usa recursos que possibilitam a reflexão a partir de oficinas em que os participantes, por meio de atividades que trazem situações-problema, fazem a exposição de vivências do cotidiano e trocam experiências com os pares.

São recursos utilizados no curso: dinâmicas de grupo, vídeos, leitura e discussão de textos em grupos menores e apresentação dos pontos discutidos para todo o coletivo, roda de conversa e atividades de dispersão.

Esses aspectos são relevantes e encontram respaldo no que MITRE et al (2008, p. 2136) pontuam sobre o assunto:

As metodologias ativas utilizam a problematização como estratégia de ensinoaprendizagem, com o objetivo de alcançar e motivar o discente, pois diante do problema, ele se detém, examina, reflete, relaciona a sua história e passa a ressignificar suas descobertas.

O uso de metodologias ativas, no contexto de ensino-aprendizagem, é necessário para o trabalho com os sujeitos que fazem uso abusivo de álcool e outras drogas, se considerarmos alguns elementos presentes na formação do profissional de saúde, tais como "a especialização precoce e um ensino dissociado do serviço e das reais necessidades do sistema de saúde vigente”. (MITRE et al., 2008, p. 2135).

Assim, ao abordar temas como as redes de atenção em saúde, a reforma psiquiátrica e a redução de danos, tomando o cotidiano e o território de atuação dos profissionais de saúde como ponto de partida, fomenta-se o aprendizado através do papel ativo do sujeito em seu meio. Tal aspecto, presente no curso "Caminhos do Cuidado", está de acordo com o proposto pelo enfoque da Educação Permanente, que preconiza:

A incorporação do ensino e do aprendizado à vida cotidiana das organizações e às práticas sociais e laborais, no contexto real em que ocorrem;

A modificação substancial das estratégias educativas, a partir da prática como fonte de conhecimento e de problemas, problematizando o próprio fazer;

Colocar as pessoas como atores reflexivos da prática e construtores do conhecimento e de alternativas de ação, ao invés de receptores; 
Abordar a equipe e o grupo como estrutura de interação, evitando a fragmentação disciplinar;

Ampliação dos espaços educativos fora da aula e dentro das organizações, na comunidade, em clubes e associações, em ações comunitárias. (BRASIL, 2009, p.44).

Ainda dentro do escopo de instrumentos da metodologia ativa, destaca-se o ensino pela problematização. Este parte da Pedagogia Crítica, de Paulo Freire, na qual "o aluno precisa ser o protagonista de seu processo de aprendizagem e ao professor cabe a tarefa de despertar a curiosidade epistemológica”.(PRADO et al.,2012, p.173).

\section{Análise do curso "Caminhos do Cuidado"}

Bordenave; Pereira (apud MITRE et al, 2008, p. 2139) utilizam o "Arco de Maguerez" para representar o ensino pela problematização. Este é composto de cinco etapas: Observação da realidade, Pontos-chave, Teorização, Hipóteses de solução e Aplicação à realidade.

Na observação da realidade, o discente expressa seu modo de perceber o mundo, a partir de uma primeira leitura do tema a ser trabalhado. Esse tema é apreendido por meio de uma observação atenta da realidade, na qual os alunos apontam os aspectos que necessitam ser desenvolvidos, participando ativamente do processo de construção do conhecimento.

No curso "Caminhos do Cuidado", a observação da realidade ocorreu a partir da apresentação de vídeos que possibilitaram uma aproximação da realidade, tais como o "Crack Crack", que problematiza os preconceitos sobre usuários de drogas e o vídeo "Nãoéoqueparece- Fora de si”, que aborda as formas de alteração da consciência usadas pela humanidade, dentre as quais o uso de substâncias psicoativas. Após assistirem aos vídeos, os alunos discutiam sobre o conteúdo apresentado.

$\mathrm{Na}$ etapa referente aos pontos-chave, o discente realiza um exame mais aprofundado, de modo a analisar e refletir sobre o assunto em questão. Trata-se da fase em que o aluno destaca os itens mais relevantes da temática apresentada e elabora o que é fundamental para compreender o problema.

No curso "Caminhos do Cuidado", a identificação dos pontos-chave foi orientada por meio dos questionamentos do tutor acerca do que havia chamado mais a atenção no material exposto, e acerca da relação do tema com as práticas de cuidado em saúde.

Dentro do rol de atividades desenvolvidas no primeiro eixo do curso, cita-se adinâmica dos prazeres como exercício que pode ser tomado como exemplo da identificação dos pontos-chave. Nessa dinâmica são feitas discussões em pequenos grupos sobre atividades 
de que as pessoas gostam de fazer, e suas consequências positivas e negativas, o que possibilitou introduzir os conceitos de prazer e risco, e sua relação com o uso de drogas, de modo a considerar a subjetividade, reduzindo a ênfase dada à substância para considerar a função que essa exerce em um dado contexto.

$\mathrm{Na}$ teorização, os alunos são levados ao questionamento dos motivos subjacentes aos problemas identificados nas fases anteriores. Assim, as informações são analisadas pelo discente de modo a contemplar aspectos práticos e teóricos do problema estudado. Nesse processo, o aluno investiga as situações visando compreender o problema em toda a sua complexidade.

No curso "Caminhos do Cuidado", a teorização ocorreu por meio da discussãoe reflexão sobre temas relativos ao uso de drogas, às políticas de saúde e àatuação profissional aliadas a vivências práticas (as atividades de dispersão), em que se buscava relacionar o conteúdo aprendido às práticas cotidianas do trabalho como ACS e ATEnf.

$\mathrm{Na}$ etapa hipóteses de solução, o discente, instrumentalizado a partir do que aprendeu na fase de teorização, enumera as opções passíveis de solucionar os problemas, buscando desenvolver alternativas para enfrentar os desafios de modo analítico e criativo, a partir da confrontação entre teoria e realidade.

No curso citado, são apresentadas as políticas públicas de atenção básica, de saúde mental e de atenção ao usuário de álcool e outras drogas. A partir da discussão sobre tais políticas, os profissionais são chamados a responder de que modo podem implementar ações de redução de danos no cotidiano de seu trabalho.

$\mathrm{Na}$ fase aplicação à realidade, os alunos colocam em prática as soluções encontradas na fase anterior e são conduzidos à construção de conhecimentos que possibilitem transformar a realidade. Nesse processo, a capacidade de generalização do conteúdo adquirido para outras situações e o discernimento sobre circunstâncias em que a aplicação das soluções encontradas não é possível são elementos apreendidos pelos discentes.

No curso "Caminhos do Cuidado", um dos objetivos propostos é que os ACS e ATEnf possam "identificar e construir coletivamente estratégias de atuação no território". (BRASIL, 2013, p. 69). Isso foi proposto a partir do conteúdo desenvolvido nas oficinas e sua relação com as práticas de cuidado no território onde os trabalhadores atuam.

Assim, a atividade ofertada para o fechamento do curso foi a apresentação de intervenções em saúde mental desenvolvidas pela equipe. Para tanto, os profissionais foram 
chamados a expor a que conclusões chegaram após executarem as atividades de dispersão. Nessa exposição, compartilharam com os pares as estratégias incorporadas ao trabalho, a partir do que foi aprendido no curso, para garantir o cuidado em saúde em mental na atenção básica e por meio da articulação de rede.

Assim, ao completar as cinco etapas "do Arco de Maguerez", os profissionais exercitam o processo ação-reflexão-ação e tornam-se capazes de construir respostas criativas a partir da realidade concreta. Isso devido às habilidades adquiridas durante a execução das atividades, em que o discente é convidado a refletir sobre os problemas apresentados e propor soluções.

\section{Desafios}

O papel ativo do discente em seu aprendizado, a análise, o debate e a reflexão sobre o tema do cuidado aos usuários de álcool e outras drogas, e a abordagem teórica e prática são potencialidades do curso. Contudo, há desafios intervenientes na capacitação dos profissionais, tais como a formação anterior, a influência de valores morais e a ambiguidade da política governamental.

Gonçalves (apud QUEIROZ, 2007) constata a existência de um perfil muito diversificado entre os trabalhadores do Programa Saúde da Família(PSF) no que tange ao preparo para lidar com a questão das drogas. Há também dificuldades objetivas, como acesso, aceitação e comunicação, e dificuldades subjetivas, como preconceitos, medo e afetos, que impactam no trabalho.

Coelho (2012) cita a culpabilização do usuário de drogas e de sua família como elemento presente no discurso dos trabalhadores, o que aponta para a reprodução de conceitos do senso comum. A autora considera que "aspectos relevantes [...] como a inserção destes sujeitos nas relações de produção e de consumo não são considerados pelos trabalhadores" ao analisar a questão do consumo de drogas. (COELHO, 2012, p. 108).

A pesquisa de mestrado de Queiroz (2007), que teve como objeto as concepções dos profissionais das equipes do Programa Saúde da Família (PSF) de Belo Horizonte sobre os usuários, o uso de drogas e os problemas relativos ao abuso e à dependência, destaca que a convivência dos profissionais do PSF com usuários de álcool e outras drogas é considerada difícil, por mais da metade dos entrevistados. 
Nessa pesquisa, foi analisado de que modo as concepções dos profissionais são articuladas a valores políticos, sociais e morais, objetivando verificar possibilidades e limites na adoção de estratégias de redução de danos. Para tal, usou-se metodologia quantitativa descritiva, com aplicação de questionário em entrevista face a face, em uma amostra de 120 profissionais de equipes de saúde da família. Os resultados apontam a potencialidade do PSF como local favorável para as ações de redução de danos, já que os profissionais aceitam a proposta, mas, também, apontam o limite já que essa aceitação coexiste com valores e práticas pautados no paradigma da abstinência.

Queiroz (2007, p.161) indica que "há uma correspondência positiva entre ser religioso ou espiritualizado e ter sentimentos negativos associados à proposta de redução de danos." Outro aspecto indicado pela pesquisa é que $85 \%$ dos profissionais entrevistados consideram que qualquer uso de substância psicoativa trará prejuízos ao usuário, o que sugere adesão a conceitos do modelo de discurso sobre drogas pautado na noção de doença.

A pesquisa de Cordeiro et al. (2014), cujo objetivo foi avaliar as mudanças nas concepções e práticas dos ACS após receberem capacitação sobre ações voltadas aos usuários de álcool e outras drogas na atenção básica, aponta que por considerar restrito o alcance de sua ação, a maioria dos ACS entrevistados “"tentava' ignorar o consumo de droga no território e/ou o naturalizava".

$\mathrm{Na}$ coleta de dados da pesquisa supracitada, utilizou-se formulário pré-pós- teste, com questões discursivas, que foram respondidas por 16 ACS. Os resultados mostraram que houve transformação nas concepções dos ACS sobre o consumo de drogas e a percepção de suas limitações no campo prático assim como a identificação da responsabilidade estatal no que tange às políticas públicas.

No que diz respeito à categoria profissional, os agentes comunitários de saúde, são, de acordo com a pesquisa de Queiroz (2007, p.161), os mais propícios à adoção da estratégia de redução de danos. A autora atribui tal postura ao fato dos ACS serem, em sua maioria, jovens, e à proximidade destes da rotina dos usuários dos serviços de saúde, o que facilitaria o vínculo.

Entretanto, o cenário não parece ser o mesmo para auxiliares e técnicos de enfermagem. Cardoso et al. (2011) apontam para a fragmentação do trabalho, visto pelos profissionais como focado na recuperação do corpo doente e nas necessidades explícitas, ou seja, aquelas relacionadas às queixas ou sintomas dos usuários. 
Cardoso et al. (2011) também afirmam que ainda que os auxiliares e técnicos de enfermagem reconheçam que há usuários que precisam conversar e procurem o serviço de saúde com esse objetivo, os profissionais não percebem tal conversa como parte do trabalho em saúde. Os autores ainda citam o acolhimento da demanda não programada como um fator que engendra conflito, medo e sofrimento nos profissionais, por não se sentirem preparados para isso.

Esses elementos apontam para a necessidade de que conceitos como vínculo e acolhimento sejam apresentados a esses trabalhadores durante a formação profissional, já que esta é, muitas vezes, voltada para o trabalho nos hospitais, e a formação para o trabalho no Programa Saúde da Família é ausente. (CARDOSO et al, 2011; OGATA; FRANÇA, 2010).

Nesse sentido, a importância da tecnologia leve é um aspecto que precisa ser trabalhado com esses profissionais:

A tecnologia leve produz-se no trabalho vivo, em ato, em um processo de relações, isto é, no encontro entre o trabalhador em saúde e o usuário/paciente. Neste momento de falas, escutas, criam-se cumplicidades, relações de vínculo, aceitação e produz-se a responsabilidade em torno do problema que vai ser enfrentado. (MERHY apud ROCHA; ALMEIDA, 2000, p.98).

Outro desafio é o fato da política governamental no Brasil, no que tange ao campo de álcool e outras drogas, mostrar-se ambígua por incorporar elementos de dois paradigmas distintos: o da redução de danos e o da abstinência.

A redução de danos pode ser entendida como "estratégia de saúde pública que visa reduzir os danos causados pelo abuso de drogas lícitas e ilícitas, resgatando o usuário em seu papel autorregulador, sem a preconização imediata da abstinência e incentivando-o à mobilização social nas ações de prevenção e de tratamento”. (BRASIL, 2003, p.27).

O paradigma da abstinência preconiza que quando se trata de drogas, o único objetivo a ser alcançado é a abstinência, já que o consumo de substâncias psicoativas é associado à criminalidade (modelo jurídico- moral) ou àdoença (modelo médico). Nesse contexto, as estratégias adotadas são a redução da oferta (por meio de ações como prisão de traficantes, apreensão de drogas e destruição de plantações) e redução da demanda (afastar o usuário das drogas, muitas vezes por meio de internações).

Enquanto a estratégia de redução de danos aposta nas ações desenvolvidas no território, por meio da articulação de redes que possibilitem ao usuário a obtenção de suporte social, sua responsabilização pelo tratamento, já que se trata de um sujeito autônomo e capaz de fazer escolhas, no paradigma daabstinência, o usuário é percebido como alguém impotente 
diante da droga, doente, criminoso, que precisa ser excluído do convívio social para ser tratado. No contexto da redução de danos, considera-se que o consumo de drogas sempre existiu como prática humana, enquanto no paradigma da abstinência busca-se o objetivo de que não haja drogas na sociedade. Portanto, são modelos que partem de pressupostos distintos quanto à concepção de sujeito, de sociedade e formas de tratamento.

No Brasil, cita-se a coexistência de dois modelos: a estratégia de redução de danos, conforme descrita na Política do Ministério da Saúde para a Atenção Integral a Usuários de Álcool e Outras Drogas, de 2003, e a aceitação de modelos que preconizam a abstinência, conforme disposto na portaria 131/2012, que trata da inclusão das Comunidades Terapêuticas na Rede de Atenção Psicossocial.

Dessa forma, o fato de modelos opostos estarem contemplados dentro da política oficial pode ser um elemento que dificulta a apreensão das especificidades de cada proposta por profissionais com formação incipiente no campo da saúde mental.

\section{Considerações Finais}

A capacitação de recursos humanos para o trabalho no Sistema Único de Saúde, citada na lei orgânica da saúde 8080/1990, é um tema presente em outras legislações brasileiras (Constituição Federal (1988), Norma Operacional Básica de Recursos Humanos para o Sistema Único de Saúde (2003), Política Nacional de Educação Permanente em Saúde (2009)).

No que tange ao campo de álcool e outras drogas, a Política para Atenção Integral a Usuários de Álcool e Outras Drogasaponta a necessidade de capacitar não só profissionais que trabalham nos CAPS AD (Centros de Atenção Psicossocial Álcool e Drogas), mas também aqueles que trabalham no Programa Saúde da Família e no Programa de Agentes Comunitários de Saúde, inclusive profissionais de nível médio. (BRASIL, 2003, p.20). Nesse sentido, a capacitação para agentes comunitários de saúde (ACS), auxiliares e técnicos de enfermagem (ATEnf) por meio do curso "Caminhos do Cuidado" visa qualificar esses profissionais na temática a partir da problematização das vivências do cotidiano de trabalho no território.

A metodologia ativa, utilizada no curso, favorece a aprendizagem por motivar o discente e fomentar o papel ativo deste na transformação da realidade. Tal metodologia é um recurso potente para profissionais de saúde, porque propicia a reflexão e a crítica acerca dos 
contextos que envolvem o uso prejudicial de drogas e instrumentaliza os trabalhadores a lidar com a complexidade das situações, por meio da apropriação de conceitos do campo da saúde mental e de sua aplicação na atenção básica.

Contudo, é imprescindível ressaltar que há limitações na proposta, uma vez que se trata de capacitação pontual, sendo necessário que o tema seja tratado por meio de atividades continuadas, não só devido à sua complexidade, mas também porque a adoção da redução de danos como política pública é relativamente recente na história do país.

No que tange à ambiguidade da política governamental, é essencial queos diferentes modelos que tratam do uso de drogas sejam contextualizados para os profissionais. Propõe-se que em capacitações sobre o temaseja abordado o histórico das políticas públicas sobre álcool e outras drogas.

Há que se considerar, também, a influência dos valores pessoais dos trabalhadores quando atuam no campo de álcool e outras drogas: como a visão moral acerca do usuário de drogas pode restringir o trabalho no campo da redução de danos. Assim, é necessário trabalhar os preconceitos, estigmas e afetos associados ao uso de drogas para que o usuário possa ser acolhido em sua singularidade.

Como se trata de capacitação iniciada em 2013, prevista para 290.197 agentes comunitários de saúde, e auxiliares e técnicos de enfermagem de todo o Brasil, sugere-se estudos futuros que possam avaliar o impacto da formação recebida na assistência ao usuário.

\section{REFERÊNCIAS}

BRASIL. Conselho Nacional de Saúde. Resolução no 330, de 4 de novembro de 2003. Aplica 'os princípios e diretrizes para a norma operacional básica de recursos humanos para o SUS (NOB/RH-SUS)' como política nacional de gestão do trabalho e da educação em saúde, no âmbito do SUS. Diário Oficial da União, Poder Executivo, Brasília, DF, 4 dez. 2003.

BRASIL. Constituição da República Federativa do Brasil. Brasília: Senado Federal, 1988.

BRASIL. Lei n.8.080 de 19 de setembro de 1990. Dispõe sobre as condições para a promoção, proteção e recuperação da saúde, a organização e o funcionamento dos serviços correspondentes e dá outras providências. Diário Oficial da União, Brasília, 1990. Seção1, p.18055-18059.

BRASIL. Ministério da Saúde. Portaria 131 de 26 de janeiro de 2012. Brasília: Ministério da Saúde, 2012. 
BRASIL. Ministério da Saúde. Secretaria de Gestão do Trabalho e da Educação na Saúde. Departamento de Gestão da Educação em Saúde. Política Nacional de Educação Permanente em Saúde / Ministério da Saúde, Secretaria de Gestão do Trabalho e da Educação na Saúde, Departamento de Gestão da Educação em Saúde. - Brasília: Ministério da Saúde, 2009.

BRASIL. Ministério da Saúde. Secretaria Executiva Coordenação Nacional de DST e AIDS. A política do Ministério da Saúde para a atenção integral a usuários de álcool e outras drogas. Brasília: Ministério da Saúde, 2003. 60 p. il. (Série B. Textos básicos de saúde).

BRASIL. Ministério da Saúde. Caminhos do Cuidado: caderno do tutor, Secretaria de Gestão do Trabalho e da Educação na Saúde; Fundação Oswaldo Cruz, Instituto de Comunicação e Informação Científica e Tecnológica em Saúde; Grupo Hospitalar Conceição, Centro de Educação Tecnológica e Pesquisa em Saúde - Escola GHC. Brasília: Ministério da Saúde, 2013. 180 p. Formação em saúde mental (crack, álcool e outras drogas) para agentes comunitários de saúde e auxiliares/técnicos em enfermagem da Atenção Básica.

BRASIL. Portal Brasil. Crack, é possível vencer: enfrentar o crack; compromisso de todos. Brasília: Governo Federal, 2013.

CARDOSO, T. Z. et al. Processo de trabalho de auxiliares e técnicos de enfermagem na atenção básica à saúde. Rev. bras. enferm., Brasília, v. 64, n. 6, p. 1087-1093, dez. 2011.

COELHO, H.V. A assistência ao usuário de drogas na atenção básica: elementos do processo de trabalho em unidade básica de saúde. 2012. 224p. Dissertação (Mestrado em Enfermagem). Escola de Enfermagem da Universidade de São Paulo.

CORDEIRO, L. et al. Avaliação de processo educativo sobre consumo prejudicial de drogas com agentes comunitários de saúde. Saúde soc., São Paulo, v. 23, n. 3, p. 897-907, set. 2014.

MITRE, S. M.et al. Metodologias ativas de ensino-aprendizagem na formação profissional em saúde: debates atuais. Ciênc. saúde coletiva, Rio de Janeiro, v. 13, supl. 2, p. 2133-2144, dez. 2008.

OGATA, M. N.; FRANCA, Y. Atuação do auxiliar de enfermagem na Estratégia Saúde da Família. Acta paul. enferm., São Paulo, v. 23, n. 4, p. 506-511, 2010.

PRADO, M. L. do et al. Arco de Charles Maguerez: refletindo estratégias de metodologia ativa na formação de profissionais de saúde. Esc. Anna Nery, Rio de Janeiro, v. 16, n. 1, p. 172-177, mar. 2012.

QUEIROZ, I.S. Adoção de ações de redução de danos direcionados aos usuários de drogas: concepções e valores de equipes do Programa de Saúde da Família. Pesquisas e Práticas Psicossociais, São João Del Rei. v. 2, n. 1, p. 152- 163, 2007.

ROCHA, S. M. M.; ALMEIDA, M. C. P.. O processo de trabalho da enfermagem em saúde coletiva e a interdisciplinaridade. Rev. Latino-Am. Enfermagem, Ribeirão Preto, v. 8, n. 6, p. 96-101, dez. 2000. 
SANTOS, J.A.T.; OLIVEIRA, M.F. Políticas públicas sobre álcool e outras drogas: breve resgate histórico. Journal of Nursing Health, Pelotas (RS), v. 1, n. 2, p. 82-93, jan./jun. 2012. 